\title{
ON THE OSCILLATION AND NONOSCILLATION OF SECOND ORDER SUBLINEAR EQUATIONS
}

\author{
MAN KAM KWONG ${ }^{1}$ AND JAMES S. W. WONG
}

\begin{abstract}
ABSTRACr. An oscillation criterion and a nonoscillation criterion are given for the sublinear equation $y^{\prime \prime}+a(t)|y|^{\gamma}$ sgn $y=0,0<\gamma<1, t \in[0, \infty)$, where $a(t)$ is allowed to change sign. When applied to the special case $a(t)=t^{\lambda} \sin t$, we deduce oscillation for $\lambda>-\gamma$ and nonoscillation for $\lambda<-\gamma$.
\end{abstract}

We are interested in determining when all continuable solutions of the sublinear Emden-Fowler equation

$$
y^{\prime \prime}(t)+a(t)|y(t)|^{\gamma} \operatorname{sgn} y(t)=0, \quad t \in[0, \infty), 0<\gamma<1,
$$

are oscillatory. We are especially motivated by the particular case $a(t)=t^{\lambda} \sin t$ or more generally $t^{\lambda} f(t)$ where $f$ is a periodic function of period $T$ such that $\int_{0}^{T} f(t) d t$ $\geqslant 0$.

We shall use as weight functions those $\phi:[0, \infty) \rightarrow[0, \infty)$ such that

$$
\phi^{\prime}>0, \quad \phi^{\prime \prime}<0 .
$$

In an earlier paper [2], the authors proved the following extension of the well-known Belohorec Theorem.

THEOREM. If there exists a function $\phi$ satisfying (2) such that

$$
\underset{T \rightarrow \infty}{\limsup } \frac{1}{T} \int_{0}^{T} \int_{0}^{t} \phi^{\gamma}(\tau) a(\tau) d \tau d t=\infty,
$$

then (1) is oscillatory, i.e. all continuable solutions of (1) are oscillatory.

An immediate consequence of this theorem is that when $a(t)=t^{\lambda} f(t)$ with $\int_{0}^{T} f(t) d t>0$ and $\lambda \geqslant-\gamma$ or with $\int_{0}^{T} f(t) d t=0$ and $\lambda>1-\gamma$, then (1) is oscillatory. When $\int_{0}^{T} f(t) d t=0$ and $\lambda \leqslant 1-\gamma$, the theorem fails to apply.

The first result of this paper is a sufficient oscillation condition that applies to cases in which

$$
\lim _{T \rightarrow \infty} \frac{1}{T} \int_{0}^{T} \int_{0}^{t} \phi^{\gamma}(\tau) a(\tau) d \tau d t
$$

exists and is finite. This condition allows us to deduce oscillation for $\lambda>-\gamma$ in our motivating example. To supplement our first result, we prove a nonoscillation

\footnotetext{
Received by the editors July 13, 1981.

AMS (MOS) subject classifications (1970). Primary 34C10, 34C 15.

Key words and phrases. Second order, nonlinear, differential equations, oscillation.

${ }^{1}$ The first author wishes to thank the Graduate School of Northern Illinois University for a grant that partially supports this research.
} 
theorem which allows us to settle the cases $\lambda<-\gamma$. The critical case $\lambda=-\gamma$ remains, however, unanswered. Thus the conjecture made by Butler in $[1, \mathrm{p} .144]$ is almost completely proved. For reference to other known results consult [2] or [3].

We define the functions

$$
\mathcal{Q}(s)=\lim _{T \rightarrow \infty} \frac{1}{T} \int_{s}^{T} \int_{s}^{t} \phi^{\gamma}(\tau) a(\tau) d \tau d t
$$

and

$$
\mathbb{Q}_{+}(s)=\max \{\mathscr{Q}(s), 0\} \text {. }
$$

THEOREM 1. If there is a weight function $\phi$ satisfying (2) so that the function $\mathbb{Q}$ given by (3) is defined and satisfies

$$
\underset{T \rightarrow \infty}{\limsup }\left(\int_{0}^{T} \frac{A_{+}^{2}(s)}{s} d s\right)\left(\int_{0}^{T} \frac{\phi^{\prime 2}(s) s}{\phi^{2}(s)} d s\right)^{-1}=\infty,
$$

then (1) is oscillatory.

Proof. As in [2], the following easily verified identity plays a crucial role:

$$
\left(\phi z^{\beta-1}\right)^{\prime \prime}+(\beta-1) \phi z^{\beta-3} z^{\prime 2}+\left(\frac{-\phi^{\prime \prime}}{\beta}\right) z^{\beta-1}=-\left(\frac{\beta-1}{\beta}\right) \phi^{\gamma} a,
$$

where $z=(y / \phi)^{\gamma}$ and $\beta=1 / \gamma>1$. Integrating (5) twice, first over $[s, t]$, then over $[s, T]$, we obtain

$$
\begin{aligned}
\phi(T) z^{\beta-1}(T) & -\phi(s) z^{\beta-1}(s)-\left(\phi(s) z^{\beta-1}(s)\right)^{\prime}(T-s) \\
& +\int_{s}^{T} \int_{s}^{t}\left(\frac{-\phi^{\prime \prime}}{\beta}\right) z^{\beta-1}(\tau) d \tau d t \\
& +(\beta-1) \int_{s}^{T} \int_{s}^{t} \phi(\tau) z^{\beta-3}(\tau) z^{\prime 2}(\tau) d \tau d t \\
= & -\left(\frac{\beta-1}{\beta}\right) \int_{s}^{T} \int_{s}^{t} \phi^{\gamma}(\tau) a(\tau) d \tau d t .
\end{aligned}
$$

Dividing by $T$ and letting $T \rightarrow \infty$, we see that, because the right-hand side tends to a limit and the integrands of the two integrals on the left-hand side as.well as the first term are nonnegative, the following limits exist and are finite:

$$
\begin{gathered}
0 \leqslant \lim _{T \rightarrow \infty} \frac{\phi(T) z^{\beta-1}(T)}{T}=K<\infty, \\
0 \leqslant \lim _{T \rightarrow \infty} \frac{1}{T} \int_{s}^{T} \int_{s}^{t} \phi(\tau) z^{\beta-3}(\tau) z^{\prime 2}(\tau) d \tau d t=G(s)<\infty
\end{gathered}
$$

and

$$
0 \leqslant \lim _{T \rightarrow \infty} \frac{1}{T} \int_{s}^{T} \int_{s}^{t}\left(-\frac{\phi^{\prime \prime}}{\beta}\right) z^{\beta-1}(\tau) d \tau d t=H(s)<\infty .
$$

It follows from (8) that

$$
\int_{0}^{\infty} \phi(\tau) z^{\beta-3}(\tau) z^{\prime 2}(\tau) d \tau<\infty
$$

since the integrand is nonnegative. 
In view of all these, (6) implies

$$
K-\left(\phi(s) z^{\beta-1}(s)\right)^{\prime}+H(s)+(\beta-1) G(s)=-\frac{\beta-1}{\beta} Q(s)
$$

and so

$$
\mathcal{Q}(s) \leqslant \frac{\beta}{\beta-1}\left(\phi(s) z^{\beta-1}(s)\right)^{\prime},
$$

from which

$$
\begin{aligned}
\frac{\mathbb{Q}_{+}^{2}(s)}{s} & \leqslant\left(\frac{\beta}{\beta-1}\right)^{2} \frac{\left(\phi(s) z^{\beta-1}(s)\right)^{\prime 2}}{s} \\
& \leqslant 2\left(\frac{\beta}{\beta-1}\right)^{2}\left[\frac{\phi^{\prime 2}(s) z^{2 \beta-2}}{s}+\frac{(\beta-1)^{2} \phi^{2}(s) z^{2 \beta-4}(s) z^{\prime 2}(s)}{s}\right]
\end{aligned}
$$

By (7) and (9),

$$
\int_{0}^{\infty} \frac{\phi^{2}(s) z^{2 \beta-4}(s) z^{\prime 2}(s)}{s} d s \leqslant K_{0} \int_{0}^{\infty} \phi(s) z^{2 \beta-3}(s) z^{\prime 2}(s) d s<\infty,
$$

where $K_{0}=\sup _{t>0} \phi(t) z^{\beta-1}(t) / t$. By (7) again

$$
\int_{0}^{T} \frac{\phi^{\prime 2}(s) z^{2 \beta-2}}{s} d s \leqslant K_{0} \int_{0}^{T} \frac{\phi^{\prime 2}(s) s}{\phi^{2}(s)} d s .
$$

Inequalities (10), (11) and (12) together contradict our hypothesis (4). This completes the proof of the theorem.

REMARK. For the case $a(t)=t^{\lambda} \sin t$ and $1>\lambda>-\gamma$, we can choose $\phi(t)=t^{\mu}$ with any $\mu$ such that $0<\mu<1$ and $1>\mu \gamma+\lambda>0$. Denote $\mu \gamma+\lambda$ by $\theta$. Then $Q$ is defined and $\mathcal{Q}(s)=s^{\theta}(\cos s+o(1))$. Since $\phi^{\prime 2}(s) s / \phi^{2}(s)=\mu^{2} / s$, (4) is satisfied and so (1) is oscillatory. The same argument works for $a(t)=t^{\lambda} f(t)$ with $\int_{0}^{T} f(t) d t=0$ and $\lambda>-\gamma$.

The following result extends the necessity part of Belohorec's Theorem, i.e. equation (1) has a nonoscillatory solution if $a(t)$ satisfies

$$
a(t) \geqslant 0, \quad \int_{0}^{\infty} t^{\gamma} a(t) d t<\infty
$$

Condition (13) implies in particular that $\lim _{T \rightarrow \infty} \int_{0}^{T} a(t) d t$ exists and is finite when $a(t)$ is nonnegative.

THEOREM 2. Suppose that $A(t)=\int_{t}^{\infty} a(t) d t$ exists for all $t \geqslant 0$. If there exists $a$ function $F(t) \in C^{1}[0, \infty)$ such that $|A(t)| \leqslant F(t)$ for all large $t$ where $F(t)=O\left(t^{-\gamma}\right)$ as $t \rightarrow \infty$ and

$$
\int_{0}^{\infty} t^{\gamma}\left|F^{\prime}(t)\right| d t=B_{0}<\infty
$$

then (1) has a nonoscillatory solution.

Proof. Let $y_{m}(t)$ be the solution of (1) satisfying $y_{m}(1)=0, y_{m}^{\prime}(1)=m$, where $m$ is a positive number. We claim that when $m$ is large enough, $y_{m}^{\prime}(t)>0$ for all $t>1$ and so $y$ is nonoscillatory. For the sake of brevity, we omit the subscript $m$ in the following discussion. 
Suppose now that $y^{\prime}(t)=0$ for some $t>1$. Let $\tau_{1}$ be the smallest of such $t$. Let $\tau_{2}$ be the smallest of all those $t$ such that $y^{\prime}(t)=2 \mathrm{~m}$. (If no such $t$ exists, let $\tau_{2}=\infty$.) Finally let $\tau=\min \left\{\tau_{1}, \tau_{2}\right\}$. Then on $[1, \tau), 0<y^{\prime}(t)<2 m$. It follows that

$$
0<y(t)<2 m t, \quad t \in[1, \tau] \text {. }
$$

At $t=\tau$, we have either

$$
y^{\prime}(\tau)=0 \quad\left(\text { if } \tau=\tau_{1}\right) \quad \text { or } \quad y^{\prime}(\tau)=2 m \quad\left(\text { if } \tau=\tau_{2}\right) .
$$

Integrating (1) once we have for $t \in[1, \tau]$

$$
y^{\prime}(t)=m-\int_{1}^{t} a(s) y^{\gamma}(s) d s .
$$

We now proceed to estimate the integral in (17) above as follows:

$$
\begin{aligned}
\left|\int_{1}^{t} a(s) y^{\gamma}(s) d s\right| & =\left|(A(1)-A(t)) y^{\gamma}(t)+\int_{1}^{t}\left(A(s)-A(1)\left(y^{\gamma}-(s)\right)\right)^{\prime} d s\right| \\
& \leqslant y^{\gamma}(t)\{2|A(1)|+|A(t)|\}+\int_{1}^{t}|A(s)|\left(y^{\gamma}(s)\right)^{\prime} d s .
\end{aligned}
$$

(The last step uses the fact that $y(t), y^{\prime}(t)>0$ on $[1, \tau)$.) We now integrate the last integral in (18) above:

$$
\left|\int_{1}^{t} A(s)\left(y^{\gamma}(s)\right)^{\prime} d s\right| \leqslant \int_{1}^{t} F(s)\left(y^{\gamma}(s)\right)^{\prime} d s \leqslant F(t) y^{\gamma}(t)+\int_{1}^{t}\left|F^{\prime}(s)\right| y^{\gamma}(\dot{s}) d s .
$$

Since $A(T)$ tends to zero as $T \rightarrow \infty$ by its very definition, $A(t)$ is bounded on $[1, \tau)$, say by a constant $K$. By assumption, there exists a constant $B_{1}$ such that $\left|t^{\gamma} F(t)\right| \leqslant B_{1}$. For $t \in[1, \tau)$, we also have from (15),

$$
\int_{1}^{t}\left|F^{\prime}(s)\right| y^{\gamma}(s) d s \leqslant(2 m)^{\gamma} \int_{1}^{t}\left|F^{\prime}(s)\right| s^{\gamma} d s \leqslant B_{0}(2 m)^{\gamma}
$$

Using (19) and (20) in (18), we find

$$
\left|\int_{1}^{t} a(s) y^{\gamma}(s) d s\right| \leqslant\left(3 K+B_{0}+B_{1}\right)(2 m)^{\gamma}=M(2 m)^{\gamma} .
$$

Substituting estimate (21) into (17), we obtain

$$
m-(2 m)^{\gamma} M \leqslant y^{\prime}(t) \leqslant m+(2 m)^{\gamma} M, \text { for all } t \in[1, \tau] \text {. }
$$

For $m>\left(2^{\gamma} M\right)^{1 /(1-\gamma)}$, we have in particular $0<y^{\prime}(\tau)<2 m$. This contradicts (16).

REMARK. For $\lambda<-\gamma$ and $a(\tau)=t^{\lambda} \sin t$, we see that $\left|\int_{t}^{\infty} a(s) d s\right|$ is less than a constant multiple of $t^{\lambda}$. Then $F(t) \equiv c t^{\lambda}$ satisfies the hypotheses of the theorem and so (1) is nonoscillatory.

Another example is offered by $a(t)=t^{-\gamma}(\log t)^{\mu} \sin t, \mu \leqslant-2$. We see that $F(t)$ can be taken to be a multiple of $t^{-\gamma}(\log t)^{\mu}$.

If $F$ is any $C^{1}$ nondecreasing function such that

$$
\int_{1}^{\infty} \frac{F(t)}{t^{1-\gamma}} d t<\infty
$$


then $F$ satisfies the hypotheses of the theorem, that is $F(t)=O\left(t^{-\gamma}\right)$, and (14) holds. To see this we apply integration by parts to obtain

$$
\frac{1}{\gamma} F(T) T^{\gamma}+\int_{1}^{T} \frac{\left[-F^{\prime}(t)\right] t^{\gamma}}{\gamma} d t=\int_{1}^{T} \frac{F(t)}{t^{1-\gamma}} d t+\frac{1}{\gamma} F(1) .
$$

Since the right-hand side is bounded, by (22), each of the terms on the left is bounded for all $T$. It can be shown by a continuity argument that the theorem still holds if $F$ satisfies (22) but no continuity requirement is assumed on $F$.

\section{REFERENCES}

I. G. J. Butler, Integral averages and the oscillation of second order ordinary differential equations, SIAM J. Math. Anal. 11 (1980), 190-200.

2. Man Kam Kwong and J. S. W. Wong, On an oscillation theorem of Belohorec, preprint.

3. J. S. W. Wong, On the generalized Emden-Fowler equation, SIAM Rev. 17 (1975), 339-360.

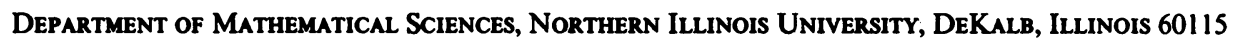
China Dyeing Works, LTD., 833 Swire House, Hong Kong

Department of MAThematics, University OF Hong Kong, Hong Kong 